VOL.1 | ISSUE 04 | ANNO 2021

DOI IN ASSEGNAZIONE

ISSN 2674-0028

\title{
TITOLO
}

\section{Management del paziente soccorso in ambiente impervio da parte dell'infermiere di emergenza territoriale: un case report.}

\section{GUARDA IL WEBINAR SU QUESTO CASE REPORT}

\section{Autori}

Enrico Lucenti, Infermiere UOC Emergenza Territoriale 118 e CTIU, Azienda USL di Piacenza. Professore a contratto corso di studio in infermieristica, Università di Parma, sede formativa di Piacenza. Direttore del Comitato Scientifico di SIIET

Cristian Sorrentino, Infermiere, Punto di Primo Intervento e 118, Ospedale di Comunità di Bobbio, Azienda USL Piacenza.

Francesco Bez, Infermiere Centrale Operativa 118 di Venezia, AULSS 3 Serenissima.

\section{INTRODUZIONE}

La valutazione del paziente ed il relativo trattamento effettuato dal primo equipaggio sanitario giunto sul luogo dell'evento hanno un'importante impatto sull'evoluzione clinica e sull'outcome del paziente traumatizzato; è necessario infatti applicare un approccio sistematico a queste situazioni guidato da raccomandazioni chiare e semplici che vedono interagire il soccorso tecnico con il soccorso sanitario ${ }_{-}^{[1]}$. 
È ben noto che il soccorso tecnico è in capo al Corpo Nazionale dei Vigili del Fuoco ${ }_{-}^{[2]}$ e al Corpo Nazionale Soccorso Alpino e Speleologico (CNSAS) ${ }_{-}^{[3]}$. E altrettanto evidente che in determinate condizioni cliniche del paziente, sebbene l'evento sia in un'ambiente impervio, il soccorso sanitario non può tardare il suo essenziale intervento ${ }_{-}^{[4]}{ }_{[5]}$.

La durata e l'esposizione a fattori ambientali per il paziente stesso convalidano

l'importanza delle figure sanitarie di emergenza territoriale anche nei luoghi impervi: il personale sanitario oltre ad avere le necessarie conoscenze per il supporto vitale dovrebbe avere competenze logistiche e sulle operazioni di soccorso ${ }_{-}^{[6]}{ }_{-}^{[7]}$.

La "Golden Hour", termine coniato nell'ormai lontano 1961, è un concetto ben noto ai soccorritori del setting pre-ospedaliero; successivamente si è vista la nascita del termine "Platinum Ten Minutes" ${ }^{\text {"[8] }}$. In entrambi i casi accorciare il tempo che trascorre dall'evento traumatico alla cura definitiva è di fondamentale importanza per un migliore esito del paziente stesso ${ }_{-}^{[9][10]}$. Esistono anche pareri contrastanti espressi in letteratura circa l'efficacia della stessa "Golden Hour" ${ }^{\text {"[11] }}$; tuttavia se vengono presi in considerazioni quei pazienti traumatizzati con patologie evidentemente tempo dipendenti (es. shock emorragico ${ }_{[12]}^{[13][} \stackrel{[14]}{\longrightarrow}$ l'importanza di un trattamento precoce torna ad essere di grande attualità â[15] $[16]$.

Questo case report descrive un soccorso in ambiente impervio dove l'infermiere di emergenza territoriale, quale primo $\mathrm{MSA}_{-}^{[17]}$ (mezzo di soccorso avanzato) giunto sul posto e quindi prima figura sanitaria, ha raggiunto il paziente sul luogo dell'evento per poterlo valutare e trattare precocemente, in piena collaborazione con il personale del soccorso tecnico giunto sul posto.

L'obiettivo è quindi quello di richiamare gli attori del sistema di emergenza territoriale all'importanza della collaborazione fra enti diversi, sanitari e tecnici non sanitari, avente chiaramente come scopo finale un più precoce ed efficace trattamento del paziente sul posto per migliorarne l'outcome.

\section{IL CASO}

- [H 4:55] Giunge la chiamata della CO 118 che richiede l'intervento del mezzo india (auto con a bordo autista soccorritore ed infermiere) per un SC01R (codice rosso traumatico in strada) con la seguente descrizione: paziente caduto da un'infrastruttura stradale da un'altezza di circa 15 metri. Viene attivato contemporaneamente un'ambulanza MSB (mezzo di soccorso di base) situato in una postazione a circa 5 
minuti dal luogo dell'evento e contestualmente i mezzi dei Vigili del Fuoco.

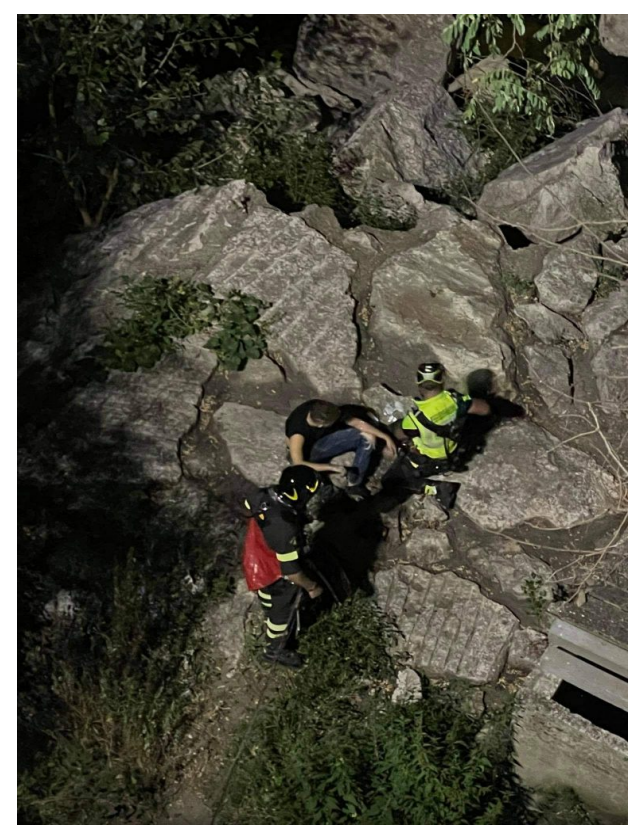

Il ferito raggiunto da VVF e Infermiere sul fondo del dirupo, lungo il corso del fiume Trebbia.

- [H 4:58] L'equipaggio india giunge sul posto dove due astanti che erano precedentemente con il paziente coinvolto fanno segno tramite le luci dei cellulari per indicare la zona dell'evento data la scarsa illuminazione artificiale. Gli astanti riferiscono che il paziente stava camminando a bordo strada (viadotto fluviale), quando per cause non note è caduto al di sotto del manto stradale (caduta di circa 15 metri) atterrando in maniera scomposta sulle rocce sottostanti adiacenti al greto di un fiume. Il paziente era in posizione seduta, vigile, agitato e fortemente dolorante. Viene avvisata immediatamente la CO 118 circa l'impossibilità immediata di raggiungere la vittima, pertanto si sollecitava l'intervento dei VVF. 


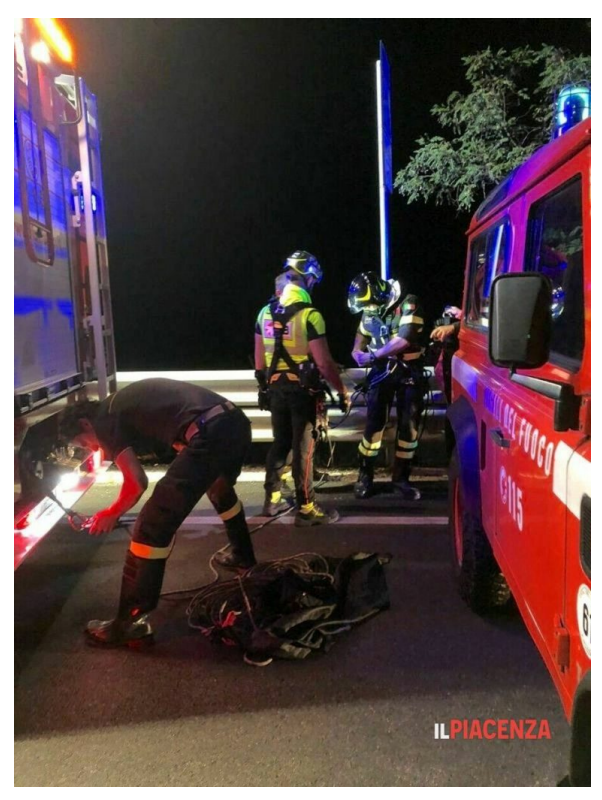

- [H 5:05] Giunge il personale del servizio tecnico ed iniziano a valutare il teatro operativo. Nel mentre l'infermiere contatta nuovamente la CO 118 per dare notizia delle prime e sommarie informazioni sanitarie riguardanti il paziente coinvolto e si decide, di comune accordo, di allertare il servizio di elisoccorso (si opta per elicottero dotato di verricello data la particolare complessità dello scenario).

- [H 5:10] Data l'evidente impossibilità di effettuare un recupero in tempi brevi, unitamente al caposquadra dei VVF, viene deciso di imbragare l'infermiere del 118 e di calarlo tramite verricello insieme ad un membro del servizio tecnico per raggiungere l'infortunato. L'infermiere prende con se lo zaino di soccorso ed il collare cervicale.

- [H 5:15] Dopo la calata, l'infermiere del 118 raggiunge la vittima, effettua l'assessment e posiziona il collare cervicale. Non essendo disponibili gli altri presidi di immobilizzazione viene effettuata la manovra di Rautek da parte del vigile del fuoco. Il paziente si presenta vigile, agitato, ma collaborante, GCS 15, vie aeree pervie.

Presenta ematoma sub-galeale in zona fronto-parietale, con escoriazioni varie al volto. Inoltre al quick look si nota frattura esposta di ulna destra, evidente deformazione dell'omero e spalla omolaterali. Viene riscontrata una saturazione del 94\%, una frequenza respiratoria di 22 atti al minuto con dolore all'emitorace destro evocato dagli atti respiratori stessi. Viene posizionato bendaggio israeliano sulla frattura esposta per arrestare il sanguinamento e proteggere la lesione. Si Rileva una pressione arteriosa di 80/50 $\mathrm{mmHg}$ con una frequenza cardiaca di 110 battiti al 
minuto, pallore cutaneo e tempo di riempimento capillare prossimo ai 2 secondi. L'infermiere posiziona una canuta venosa periferica al braccio destro con calibro 14 Gauge.

- [H 5:25] Tramite contatto radio con il resto degli operatori sul viadotto si organizza un sistema di corde per il passaggio dei presidi e vengono cosi fatti scendere barella spinale con relativo fissaggio e liquidi caldi. Nel frattempo l'infermiere rileva un ridotta captazione dei rumori respiratori all'apice destro con ridotta espansione e forte dolore alla minima palpazione. Inoltre è presente dolorabilità alla palpazione in ipocondrio destro. Viene iniziata terapia infusiva con Ringer lattato caldo $500 \mathrm{ml}$.

- [H 5:40] Il paziente viene posizionato su tavola spinale. Data l'estrema dolorabilità dell'arto superiore destro (VNS 10/10) si attua protocollo infermieristico di analgesia e si somministra Perfalgan $1 \mathrm{gr}$ ev (non si opta per la morfina per sussistenza di quadro ipotensivo). Si applica la stecco-benda a depressione per mantenere in posizione antalgica il braccio. Inoltre, valutata la dinamica, nel sospetto di emorragie interne data la positività dell'indice di shock, l'infermiere somministra 2 fiale di Acido Tranexamico come da protocollo. Si completa isolamento termico con metallina e lenzuolo.

- [H 6:00] Dopo circa un'ora dall'arrivo sul posto delle squadre di soccorso il paziente si presenta vigile ma con tendenza ad assopirsi, GCS 14, a tratti reiterante nell'eloquio, lieve miglioramento della sintomatologia dolorosa (VNS 7/10), PA 100/60 dopo infusione di $\mathbf{1 0 0 0} \mathbf{~ m l}$ totali di Ringer lattato, tempo di riempimento capillare $1 \mathrm{sec}$, FC 94, saturazione O2 94\%, FR 20.

- [H 6:10] Giunge sul luogo dell'evento l'elisoccorso con sbarco del personale sulla superficie stradale. Nel mentre il servizio di soccorso alpino ha allestito il sistema di discesa per il personale elitrasportato che viene così calato per raggiungere il pz.

- [H 6:20] Si decide per il recupero del paziente con verricello. Il medico rianimatore somministra una fiala di Fentanil per analgesia efficace e si procede all'imbrago su device specifico per estrazione aerea. Si opta per non drenare il PNX data la stabilità emodinamica e respiratoria (mantiene respiro spontaneo).

- [H 7:00] Vengono concluse le operazioni di soccorso con la partenza dell'elisoccorso per la rianimazione el centro HUB regione di riferimento.

\section{DISCUSSIONE}

Il caso sopra descritto accade in un luogo impervio ed il paziente si trova evidentemente in una condizione tale per cui è difficile raggiungerlo nell'immediato. Come si può evincere dalla time line riportata, dall'arrivo sul posto delle equipe di soccorso al momento in cui il 
paziente decolla per il centro Hub di riferimento trascorrono circa 2 ore; a queste ore si deve aggiungere poi il tempo di volo. Il paziente si è presentato fina da subito come un politrauma a dinamica maggiore con evidenti segni di instabilità.

Il riconoscimento precoce dell'instabilità clinica può essere difficile ${ }_{-}^{[18]}$ soprattutto in ambienti come il sopra descritto ed è noto che un ritardo nell'individuazione del peggioramento dei sintomi è correlato ad un ritardo nell' attivazione di un livello più elevato di assistenza ${ }_{-}^{[19]}$. Gli infermieri sono tra i professionisti con le competenze idonee a rilevare precocemente i segni di instabilità clinica $\stackrel{[20]}{[21]}$.

Applicando la "Modified Early Warning Score" (MEWS ${ }^{[22]}$ [23] coi parametri rilevati al paziente, alle ore 5:15, alla prima valutazione parametrica da parte dell'infermiere, si ottiene un punteggio di 5 (senza sommando il valore numerico attribuito dalla misurazione della temperatura corporea). È bene ricordare che attraverso la MEWS il paziente è definito critico e quindi instabile con un punteggio pari o superiore a 4 . La scelta di calare il personale sanitario (infermiere mezzo india) ha permesso di avere un'inquadramento globale e precoce circa il potenziale rischio evolutivo del paziente. Dopo la stabilizzazione del paziente ed il trattamento per quanto fosse possibile, il punteggio della MEWS è sceso a 2 . Anche in questo caso manca la sommatoria del valore attribuito dalla rilevazione della temperatura corporea ma si è voluto riportare il dato comunque al fine di valutarne il trend in discesa (indici stabilizzazione emodinamica).

Utilizzando il ben noto indice di shock nella prima valutazione il paziente presenta un valore pari a 1,37, successivamente, nella seconda valutazione, dopo comunque aver avuto la possibilità di ricevere dei trattamenti, il paziente presenta un indice di shock pari a 0,94, mostrando quindi un possibile miglioramento dal punto di vista della stabilità emodinamica. A tal proposito conviene ricordare che un indice di shock $>$ di 0,7 è già di per se predittivo di un tasso di mortalità in aumento anche se l'indice stesso non dovrebbe essere mai utilizzato da solo ${ }^{[24]}{ }_{[25]}$ ma in correlazione agli altri parametri vitali, all'esame obiettivo, alla dinamica e alla cinematica del trauma.

È doveroso ricordare, nella trattazione di un caso simile, che la sicurezza degli operatori deve essere preponderante nelle scelte attuative del soccorso stesso. Raggiungere il paziente in condizioni di non sicurezza non sarebbe stato ne auspicabile ne eticamente corretto. Avere permesso all'infermiere del mezzo di india di calarsi sul luogo dell'evento e raggiungere precocemente il paziente, rappresenta una scelta ponderata ma soprattutto una scelta presa fra professionisti appartenenti a profili professionali diversi ma aventi tutti in comune lo scopo ultimo: portare soccorso alla vittima. 


\section{CONCLUSIONE}

In alcune circostanze non sempre è possibile prestare le prime cure essenziali al paziente critico specie se lo stesso si trova in luogo impervio, luogo difficilmente raggiungibile nell'immediato da parte dei soccorritori. Chi opera sul territorio deve conoscere il proprio territorio e le peculiarità del soccorso che si vengono a creare a seconda della variabile ambientale. Tuttavia, nell'insieme delle patologie tempo dipendenti e della gravità del trauma, prima viene portato soccorso al paziente e meglio è in virtù del suo outcome. Ancor meglio se il soccorso che viene portato sul campo, oltre che a quello di base, prevede un'assessment ed un management avanzato. Qui si inserisce la figura dell'infermiere del sistema di emergenza territoriale che, debitamente formato, e in collaborazione con le figure professionali preposte per la gestione dello scenario in sicurezza, è in grado di portare competenze avanzate a supporto del paziente anche in situazioni ambientali difficili. Una risorsa sanitaria presente sul luogo dell'evento non può solo restare a guardare ma deve essere uno fra i principali attori a scendere in campo precocemente. Questo caso evidenzia il miglioramento del paziente trattato precocemente grazie alla calate dell'infermiere del 118 vicino alla vittima. Tuttavia è auspicabile una più ampia collaborazione a livello di pianificazione formativa fra enti diversi ma accomunati da un unico fattore: il fattore umano, cioè il paziente da soccorrere.

\section{REFERENZE}

${ }^{[1]}$ Popa TO. Prehospital Emergency Care in Acute Trauma Conditions [Internet]. Available at: https://www.intechopen.com/chapters/67641 (Consulted: 6/10/2021).

${ }^{[2]}$ Dipartimento dei Vigili del fuoco, del Soccorso pubblico e della Difesa civile [Internet]. Available at:

https://www.interno.gov.it/it/ministero/dipartimenti/dipartimento-dei-vigili-fuoco-soccorso-p ubblico-e-difesa-civile (Accessed: 6/10/2021)

${ }^{[3]}$ Corpo Nazionale Soccorso Alpino e Speleologico [Internet]. https://www.cnsas.it/attivita/ (Accessed: 6/10/2021).

${ }^{[4]}$ Hearns S. The Scottish mountain rescue casualty study. Emerg Med J. 2003 May;20(3):281-4. doi: 10.1136/emj.20.3.281. 
${ }^{[5]}$ Strapazzon G, Reisten O, Argenone F, Zafren K, Zen-Ruffinen G, Larsen GL, Soteras I. International Commission for Mountain Emergency Medicine Consensus Guidelines for OnSite Management and Transport of Patients in Canyoning Incidents. Wilderness Environ Med. 2018 Jun;29(2):252-265.

${ }^{[6]}$ Soteras I, Subirats E, Strapazzon G. Epidemiological and medical aspects of canyoning rescue operations. Injury. 2015 Apr; 46(4):585-9.

${ }^{\text {[7] }}$ Ströhle M, Beeretz I, Rugg C, Woyke S, Rauch S, Paal P. Canyoning Accidents in Austria from 2005 to 2018. Int J Environ Res Public Health. 2019 Dec 22;17(1):102.

${ }_{-}^{[8]}$ Calland V. Extrication of the seriously injured road crash victim. Emerg Med J. 2005 Nov;22(11):817-21.

[9] Okada K, Matsumoto H, Saito N, Yagi T, Lee M. Revision of 'golden hour' for hemodynamically unstable trauma patients: an analysis of nationwide hospital-based registry in Japan. Trauma Surg Acute Care Open. 2020 Mar 10;5(1):e000405.

${ }^{[10]}$ Dinh MM, Bein K, Roncal S, Byrne CM, Petchell J, Brennan J. Redefining the golden hour for severe head injury in an urban setting: the effect of prehospital arrival times on patient outcomes. Injury. 2013 May;44(5):606-10.

[11] Schroeder PH, Napoli NJ, Barnhardt WF, Barnes LE, Young JS. Relative Mortality Analysis Of The "Golden Hour": A Comprehensive Acuity Stratification Approach To Address Disagreement In Current Literature. Prehosp Emerg Care. 2019 Mar-Apr;23(2):254-262.

${ }^{[12]}$ Soudry E, Stein M. Prehospital management of uncontrolled bleeding in trauma patients: nearing the light at the end of the tunnel. Isr Med Assoc J. 2004 Aug;6(8):485-9.

${ }^{[13]}$ Almuwallad A, Cole E, Ross J, Perkins Z, Davenport R. The impact of prehospital TXA on mortality among bleeding trauma patients: A systematic review and meta-analysis. J Trauma Acute Care Surg. 2021 May 1;90(5):901-907.

${ }^{[14]}$ Braverman MA, Smith A, Pokorny D, Axtman B, Shahan CP, Barry L, Corral H, Jonas RB, Shiels M, Schaefer R, Epley E, Winckler C, Waltman E, Eastridge BJ, Nicholson SE, Stewart RM, Jenkins DH. Prehospital whole blood reduces early mortality in patients with hemorrhagic shock. Transfusion. 2021 Jul;61 Suppl 1:S15-S21.

${ }^{[15]}$ Tucker H, Avery P, Brohi K, Davenport R, Griggs J, Weaver A, Green L. Outcome 
measures used in clinical research evaluating pre-hospital blood component transfusion in traumatically injured bleeding patients: A systematic review. J Trauma Acute Care Surg. $2021 \mathrm{Jul} 12$.

${ }^{[16]}$ Pierrie SN, Seymour RB, Wally MK, Studnek J, Infinger A, Hsu JR; Evidence-based Musculoskeletal Injury and Trauma Collaborative (EMIT). Pilot randomized trial of prehospital advanced therapies for the control of hemorrhage (PATCH) using pelvic binders. Am J Emerg Med. 2021 Apr;42:43-48.

[17] Ministero Della Salute. I mezzi di soccorso [Internet]. Available at:

https://www.salute.gov.it/portale/temi/p2_6.jsp?id=1100\&area=118\%20Pronto\%20Soccorso $\&$ menu=vuoto (Accessed: 6/10/2021).

${ }^{[18]}$ Gardner-Thorpe J, Love N, Wrightson J, Walsh S, Keeling N. The value of Modified Early Warning Score (MEWS) in surgical in-patients: a prospective observational study. Ann R Coll Surg Engl. 2006 Oct;88(6):571-5.

${ }^{[19]}$ Dalton M, Harrison J, Malin A, Leavey C. Factors that influence nurses' assessment of patient acuity and response to acute deterioration. Br J Nurs. 2018 Feb 22;27(4):212-218.

${ }^{[20]}$ Jackson S, Penprase B, Grobbel C. Factors Influencing Registered Nurses' Decision to Activate an Adult Rapid Response Team in a Community Hospital. Dimens Crit Care Nurs. 2016 Mar-Apr;35(2):99-107.

[21] Liaw SY, Scherpbier A, Klainin-Yobas P, Rethans JJ. A review of educational strategies to improve nurses' roles in recognizing and responding to deteriorating patients. Int Nurs Rev. 2011 Sep;58(3):296-303.

[22] Subbe CP, Kruger M, Rutherford P, Gemmel L. Validation of a modified Early Warning Score in medical admissions. QJM. 2001 Oct;94(10):521-6.

${ }^{[23]}$ Beretta M, Popolla A, Subbe CP, Cornelli S, Bolzoni M, Grossi CF, Capelli P, Lucenti E, Guasconi M, Granata C. "Modified Early Warning Score" (MEWS) per il riconoscimento precoce dell'instabilità clinica. Scenario 2021; 38 (3): 5-9 IN PRESS.

${ }^{[24]}$ Koch E, Lovett S, Nghiem T, Riggs RA, Rech MA. Shock index in the emergency department: utility and limitations. Open Access Emerg Med. 2019 Aug 14;11:179-199.

${ }^{[25]}$ Birkhahn RH, Gaeta TJ, Terry D, Bove JJ, Tloczkowski J. Shock index in diagnosing early 
acute hypovolemia. Am J Emerg Med. 2005 May;23(3):323-6. 